Center, Tokyo, Japan; 'Department of Surgery, Nagano Matsushiro General Hospital, Nagano, Japan; ${ }^{3}$ Nikkei Disease Prevention Center, São Paulo, Brazil

Introduction Previous studies have shown higher breast cancer incidence and mortality among Japanese Brazilians than Japanese. To clarify the difference in hormone levels among populations, we compared postmenopausal endogenous sex hormone levels among Japanese living in Japan, Japanese Brazilians living in São Paulo, and non-Japanese Brazilians living in São Paulo.

Methods A cross-sectional study was conducted using a control group of case-control studies in Nagano, Japan and São Paulo, Brazil. Subjects were postmenopausal women aged over 55 years old who provided blood samples. We measured oestradiol, oestrone, androstenedione, dehydroepiandrosterone sulphate (DHEAS), testosterone and free testosterone by radioimmunoassay, bioavailable oestradiol by the ammonium sulphate precipitation method, and sex-hormone binding globulin (SHBG) by immunoradiometric assay. A total of 363 women were included for the present analyses: 185 Japanese, 44 Japanese Brazilians and 134 non-Japanese Brazilians.

Results Japanese Brazilians had significantly higher levels of oestradiol, bioavailable oestradiol, oestrone, testosterone, and free testosterone, and lower SHBG levels than Japanese. Japanese Brazilians also had significantly higher levels of bioavailable oestradiol, oestrone, and DHEAS, and lower levels of SHBG and androstenedione than non-Japanese Brazilians. Levels of oestradiol, testosterone, and free testosterone, however, did not differ between Japanese Brazilians and non-Japanese Brazilians. These differences were observed even after adjustment for known breast cancer risk factors.

Conclusions We found higher levels of oestrogens and androgens in Japanese Brazilians than in Japanese, and similar to or higher levels than in non-Japanese Brazilians. Our findings may help explain the increase in incidence and mortality of breast cancer among Japanese Brazilians.

\section{P1-447 HOW LONG DO PATIENTS IN THE UK GET TREATED FOR NON-SPECIFIC RESPIRATORY SYMPTOMS BY GENERAL PRACTITIONERS BEFORE THEY ARE DIAGNOSED WITH LUNG CANCER?}

doi:10.1136/jech.2011.142976g.37

B Iyen-Omofoman, ${ }^{*}$ L Tata, R Hubbard. University of Nottingham, Nottingham, UK

Introduction Most people with lung cancer in the UK are diagnosed late when curative treatment is no longer an option. This research aimed to determine the pattern of symptom reporting to GPs before lung cancer diagnosis to establish whether there is potential for developing a scoring system to allow cases to be diagnosed earlier.

Methods We used data from The Health Improvement Network (THIN) - A computerised longitudinal primary care database. We identified 12121 incident cases of lung cancer diagnosed between 2000 and 2009 and matched each case with up to four controls by age, sex and general practice $(n=48216)$. Conditional logistic regression was carried out to estimate the ORs for symptoms 2 years before and 1 year before lung cancer diagnosis in cases compared to controls.

Results A sharp increase in the consultation frequency among lung cancer cases was found for cough, haemoptysis, chest/shoulder pain, voice hoarseness, dyspnoea, weight loss, lower respiratory tract infections, non-specific chest infections and chronic obstructive airway disease, about 9 months before they were diagnosed with lung cancer. The highest $\mathrm{OR}$ in cases compared to controls at 1 year was noted for haemoptysis (OR 54.7, 95\% CI 42.5 to 70.4) and the lowest was for upper respiratory tract infections (OR 1.72, 95\% CI 1.55 to 1.92$)$.
Conclusions Patients with lung cancer have an increase in reporting of symptoms to the GP which occurs about 9 months before they are diagnosed. Future work will aim to develop these results into a predictive score to allow earlier diagnosis of lung cancer.

\section{P1-448 USING A SOCIAL MARKETING TOOL TO IDENTIFY SECTORS OF THE UK WHERE LUNG CANCER INCIDENCE IS HIGHEST}

doi:10.1136/jech.2011.142976g.38

B Iyen-Omofoman, ${ }^{*}$ L Tata, R Hubbard. University of Nottingham, Nottingham, UK

Introduction To target lung cancer awareness campaigns and increase earlier ascertainment of disease, there is a need to identify the sectors of society most at risk of developing lung cancer. Using Experian's Mosaic social marketing tool (a consumer classification of all UK households and postcodes into 61 groups and 11 types), we aimed to identify the UK populations with the highest incidence of lung cancer.

Methods All incident cases of lung cancer from 2000 to 2009 in a UK computerised primary care database were identified. Lung cancer incidence rates were stratified by 3 -yearly calendar periods, age, sex, socioeconomic status, UK health authority and Mosaic groups and types.

Results A total of 12135 incident cases of lung cancer were identified. Overall incidence of lung cancer was 41.4 per 100000 personyears and this increased with increasing deprivation. The highest incidence of lung cancer was in the North-West of England and the lowest rate was in London. The Mosaic types with the highest incidence of lung cancer were F39 (dignified dependency), I48 (old people in flats) and I50 (cared-for pensioners).

Conclusions Using Experian Mosaic's social marketing tool, we have identified wide variations in the incidence of lung cancer, larger than variations by socioeconomic status alone. We have also been able to determine the sectors of UK society and therefore postcodes and neighbourhoods with the highest incidence rates of lung cancer. Application of this knowledge will enable accurate targeting of media campaigns on lung cancer and also aid earlier ascertainment of lung cancer cases.

\section{P1-449 EFFECT OF STONE DUST ON LUNG AND LUNG FUNCTION TESTS OF STONE OUARRY WORKERS IN SRI LANKA}

doi:10.1136/jech.2011.142976g.39

${ }^{1,2} \mathrm{C}$ V M Jinadasa, ${ }^{*}{ }^{1} \mathrm{P}$ Tharanga, ${ }^{1} \mathrm{~N}$ Gunarathne, ${ }^{1} \mathrm{D}$ Wijerathne, ${ }^{1} \mathrm{~K}$ Lankathilaka. ${ }^{1}$ Facutly of Medicine, University of Colombo, Colombo, Sri Lanka; ${ }^{2}$ Sri Jayawardhanapura Hospital, Colombo, Sri Lanka

Introduction Stone quarry workers are constantly exposed to crystalline silica dust of different particle sizes in different concentrations. General objective To determine the effects of stone dust on respiratory health of stone quarry workers in Sri Lanka.

Methodology Respiratory symptoms of quarry workers $(n=79)$ from different sections of industry were assessed followed by a clinical examination. Spirometric indices were recorded prior to a Monday work shift and air borne dust levels were measured.

Results Workers had a mean work duration of 53 months and an average of 4.2 pack years of smoking. Regarding the respiratory symptoms, $10.1 \%$ and $11.4 \%$ workers complained of morning and nocturnal cough, respectively. While $2.5 \%$ workers had Haemoptysis and $30.4 \%$ workers complained of wheezing, $30.4 \%$ and $5.1 \%$ had Grade II and III dyspnoea respectively. One subject showed evidence of fibrosis on clinical examination. Lung function tests showed a restrictive pattern in 24 (30.4\%) workers and among them 16 had above normal exposure to dust. Obstructive pattern is seen in 9 